NBSIR 75-793

\title{
Microsecond-Resolution Pulse Calorimetry for Semiconducting Materials at High Temperatures (A Feasibility Study)
}

A. Cezairliyan and C. W. Beckett

Physical Chemistry Division Institute for Materials Research National Bureau of Standards

Washington, D. C. 20234

October 1975

Report for Contract No. 31-109-38-3174

May 1975 - June 1975

\section{Prepared for}

Argonne National Laboratory

QC gonne, Illinois 60439

100

.456

n. $75 \cdot 793$

1975 



\section{MICROSECOND-RESOLUTION PULSE CALORIMETRY FOR SEMICONDUCTING MATERIALS AT HIGH TEMPERATURES (A FEASIBILITY STUDY)}
A. Cezairliyan and C. W. Beckett

Physical Chemistry Division Institute for Materials Research National Bureau of Standards

Washington, D. C. 20234

\section{October 1975}

Report for Contract No. 31-109-38-3174

May 1975 - June 1975

Prepared for

Argonne National Laboratory

Argonne, lllinois 60439

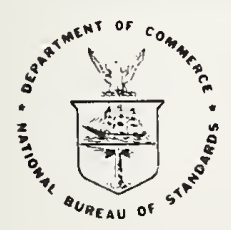

U.S. DEPARTMENT OF COMMERCE, Rogers C.B. Morton, Secretary James A. Baker, III, Under Secretary Dr. Betsy Ancker-Johnson, Assistant Secretary for Science and Technology NATIONAL BUREAU OF STANDARDS, Ernest Ambler, Acting Director 
. 
1. Introduction ................... 1

2. Description of the Method ............ 2

3. General Design Considerations . . . . . . . . 6

3.1. Electrical Circuit Equations . . . . . . . . 6

3.2. Consideration of Various Phenomena . . . . . . 9

3.2.1. Specimen stability-gravitational ... 9

3.2.2. Internal magnetic forces........ 9

3.2.3. External magnetic forces........ 10

3.2.4. Skin Effect .............. 11

3.2.5. Temperature distribution in the 12

3.2.6. Evaporation ............. 13

3.2.7. Electrical breakdown in gases . . . . 14

3.2.8. Ionization ............ 15

3.2.9. Thermionic emission ........ 16

3.2.10. Other phenomena ......... 16

4. Measurement of Experimental Quantities . . . . . 18

4.1. Power Measurements . . . . . . . . . 18

4.2. Temperature Measurements . . . . . . . . 22

4.3. Other Measurements and Diagnostic Techniques . . 25

4.4. Recording of Experimental Quantities . . . . . 27

5. Special Considerations In Relation to $\mathrm{UO}_{2}$...... 29

5.1. Heating of $\mathrm{UO}_{2}$. . . . . . . . . . . . . 29

5.2. Various Phenomena Related to $\mathrm{UO}_{2}$. . . . . . 32

5.3. Measurements on $\mathrm{UO}_{2}$. . . . . . . . . . . 37

6. Summary and Conclusions ............ . . 41

7. References . . . . . . . . . . . . . 45 



\section{Abstract}

A feasibility study is conducted for the applicability of microsecond-resolution pulse heating techniques to the measurement of selected thermodynamic properties of semiconducting substances at high temperatures. The method of pulse heating of the specimen using capacitor discharge techniques is described and general design. considerations, including electrical circuitry and various physical, electrical and chemical phenomena, are presented. Measurements of experimental quantities, such as current, vol tage, temperature and pressure are described. The special problems in relation to measurements of heat capacity and vapor pressure of uranium dioxide in the temperature range 2000 to $6000 \mathrm{~K}$ are discussed. It is concluded that the capacitor discharge circuitry and the measurement of the experimental quantities, although difficult, are not likely to present major problems. However, problems are likely to stem from the low electrical conductivity of the specimen. The experimental chamber must withstand high pressures, high voltages, with optical windows and possibly a furnace. The nature of the problems are discussed and the performance of some preliminary experiments are suggested. 


\section{Introduction}

Experimental investigations reported during the last two decades in connection with exploding wires [1] and recently more quantitative measurements on good conductors [2-7] have suggested the potential of rapid (submillisecond) heating techniques as means of measuring selected thermodynamic and transport properties of solid and liquid substances at high temperatures (above $3000 \mathrm{~K}$ ), where conventional techniques become difficult or impossible to use. The efforts reported in the literature were mostly exploratory and preliminary in nature and were in connection with measurements on metallic materials or substances which are electrically good conductors at room temperature.

This report summarizes the results of a feasibility study for the development of a transient (microsecond resolution) system for the measurements of selected thermodynamic and transport properties of solid and liquid semiconducting substances (with special emphasis on $\mathrm{UO}_{2}$ ) in the temperature range 2000 to $6000 \mathrm{~K}$.

The report is arranged to include: (a) the general description of the transient method, (b) general design considerations to include various physical, electrical, mechanical and chemical phenomena associated with transient experiments, (c) measurement of experimental quantities, such as current, voltage, temperature, pressure in transient experiments and performance of diagnostic measurements, (d) special considerations in relation to $\mathrm{UO}_{2}$ specimens, and (e) summary and conclusions. 


\section{Description of the Method}

The method is based on resistive self-heating of the specimen by the passage of high currents through it and measuring the pertinent quantities with appropriate time resolution. In general, the required quantities are: current through the specimen, potential difference across the specimen, and specimen temperature. Depending on the nature of the experiment, additional quantities (electrical and thermal, such as pressure) may be required.

Basically, the measurement system consists of an electrical powerpulsing circuit and associated high-speed measuring circuits. The pulsing circuit includes the specimen in series with an electric pulse power source (such as a capacitor bank), an adjustable resistance, a standard resistance and a fast-acting switch. The specimen is contained in a controlled-environment chamber. The high-speed measuring circuits include detectors and recording system.

Some of the electrical power pulsing circuits and the instrumentation required in a thermodynamic measurement system may be simflar to those used in exploding conductor or simflar research. A variety of such capacitive energy storage and discharge systems were developed in the 1950 s and 1960s for various applications. Their capacity ranged from joules to megajoules, and their discharge duration ranged from nanoseconds to milliseconds. In recent years, only minor changes have taken place in such systems. 
As of the present time, capacitive storage and discharge systems have been developed for reliable and almost routine operation at voltages as high as $30 \mathrm{kV}$. In spite of the difficulties that are encountered above this voltage, a few systems have been developed and have been operated at several hundred kilovolts.

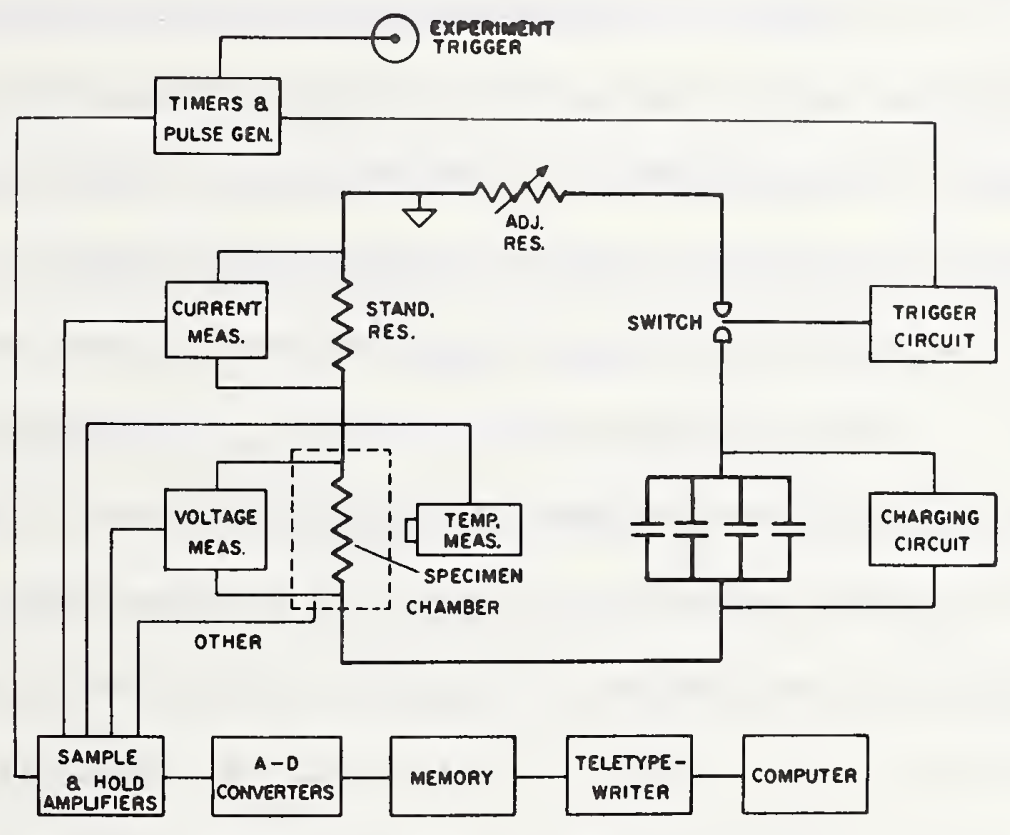

Figure 1. Functional diagram of a capacitor discharge system for the rmodynamic measurements.

A functional diagram of a typical system that may be used for thermodynamic measurements is shown in Figure 1. The pulse-power source is a capacitor bank. The charging circult includes a conventional high voltage d.c. supply (usually with 1 to $100 \mathrm{~mA}$ current 
rating), an electrostatic voltmeter, and a high-voltage switch for disconnecting the supply from the main circuit prior to the discharge. The capacitors used in such systems are designed to have very low inductance ( $I$ to $100 \mathrm{nH}$ ). The majority of the capacitors used and reported in the literature fall in the 1 to $15 \mu \mathrm{F}$ range with voltage ratings from 5 to $30 \mathrm{kV}$. Energy stored in a capacitor is expressed as $\mathrm{U}=\mathrm{CE}^{2} / 2$, where $\mathrm{C}$ is capacitance, and $\mathrm{E}$ is the voltage. Since energy depends on the square of the voltage, higher energy discharges are achieved more efficiently by increasing the charging vol tage. Because of their high current capacity and simplicity, spark gaps are frequently used as switching devices in capacitor discharge systems. A variety of types are reported in the literature. The type most commonly used for voltages up to $50 \mathrm{kV}$ consists of two highcurrent electrodes separated by a gap (air or other gas). Closing of the switch is achieved by a triggering circuit which ionizes the gas in the gap. As a result of ionization, resistance of the gap decreases to a negligible leve1, thereby causing inftiation of the discharge. Several techniques may be used to ionize the gas. The most commonly used method is to supply a relatively low energy but high voltage pulse to a trigger electrode located near or coaxial with one of the main electrodes. The resultant spark between the trigger and one of the main electrodes is sufficient to ionize the gas. Another method is to expose the gas to the radiation from a pulsed laser. Above $40 \mathrm{kV}$, the switching of high currents becomes more 
difficult. It has been found that gas-type spark gaps are not reliable due to their sensitivity to gas composition (primarily to moisture content). Vacuum type switches, composed of two electrodes placed in a vacuum container, have shown higher reliability. In this case, switching is achieved by supplying a low-energy, high-voltage pulse to one of the electrodes in such a way that the net potential difference between the two electrodes exceeds the natural breakdown vol tage.

Standard resistances, which in discharge work are sometimes referred to as "shunts", have a special coaxial configuration to reduce undesirable effects of high electromagnetic fields. Details regarding design and operation of current and voltage measuring devices as well as temperature measuring equipment are given in Section 4.

Depending on the experimental procedure and requirements, the specimens initially may be in the form of a wire, rod, tube or a composite made of a tube filled in the middle with the specimen in solid or liquid form.

Recording of experimental quantities are generally made with oscilloscopes. However, recent advances in the electronics field permit the development of systems for digital recording of quantities with microsecond resolution and with full-scale resolution of approximately 0.1 percent. 


\section{Gene ral Design Conside rations}

\subsection{Electrical Circuit Equations}

The capacitor discharge system described in the previous section and shown schematically in Figure 1 can be represented electrically as a series RLC circuit. Dynamic characteristics of such a circuit may be represented by the following equation:

$$
L \frac{d^{2} i}{d t^{2}}+R \frac{d i}{d t}+\frac{i}{C}=0
$$

where, $i$ is current, $t$ is time, and $R, L, C$ are resistance, inductance, and capacitance of the entire circult, respectively. The solution of Equation (1) is given in Table 1 for three different cases. The results are also represented graphically in Figure 2.

It should be considered that in the solutions of Equation (1), the quantities $\mathrm{R}, \mathrm{L}$, and $\mathrm{C}$ are considered to be constant. In an actual capacitor discharge experiment this assumption is reasonably satisfied for $\mathrm{L}$ and $\mathrm{C}$. However, $\mathrm{R}$ varies as a function of time because of the change in specimen resistance during heating. The degree of departure of actual results from those given in Table 1 depends on the relative magnitude of the change in specimen resistance with respect to the resistance of the entire circuit.

Critical damping is the most desirable case to have in a discharge experiment. However, because of various conflicting design and operational parameters in an actual system, this is very difficult to achieve. In 


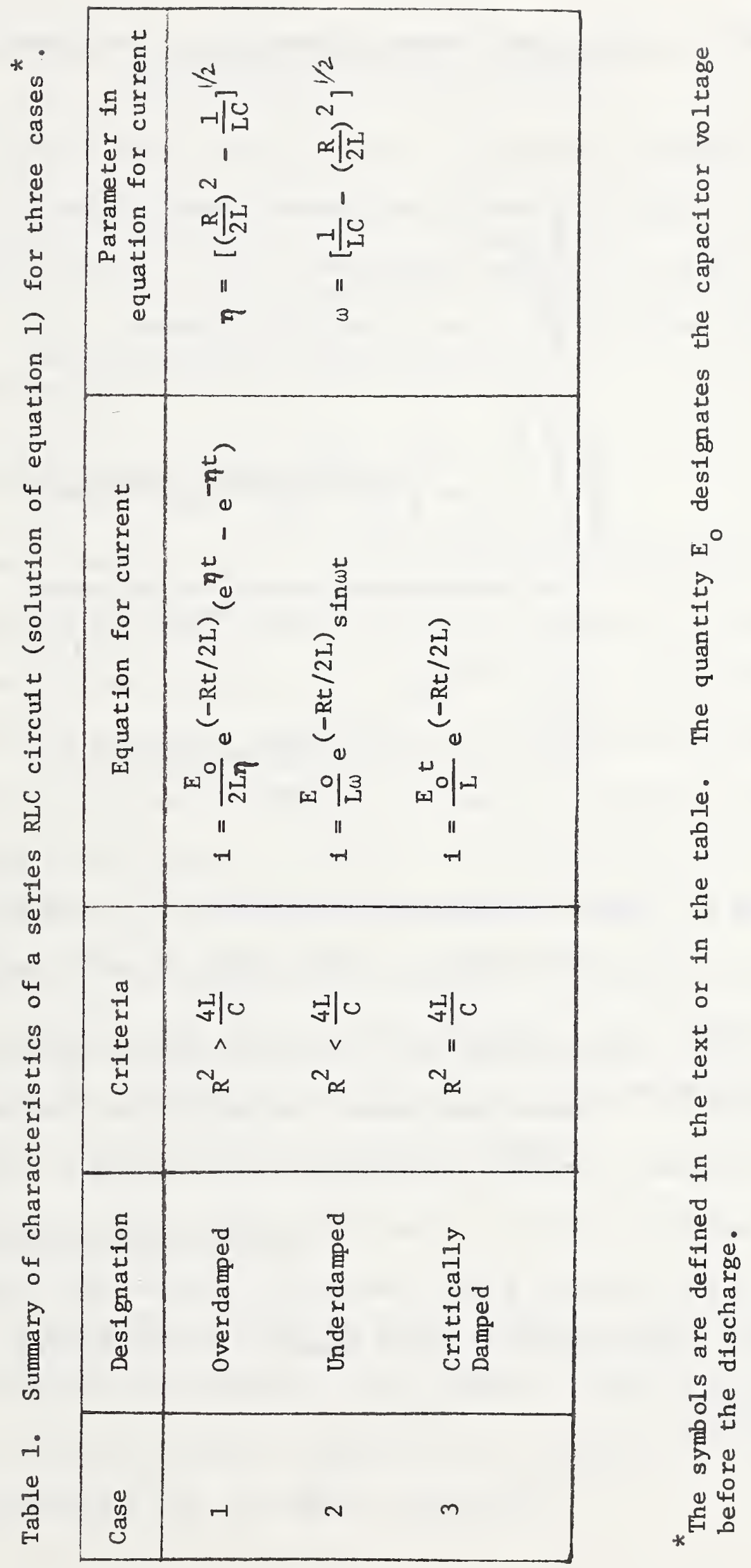


general, a typical performance corresponds to the "underdamped" case,
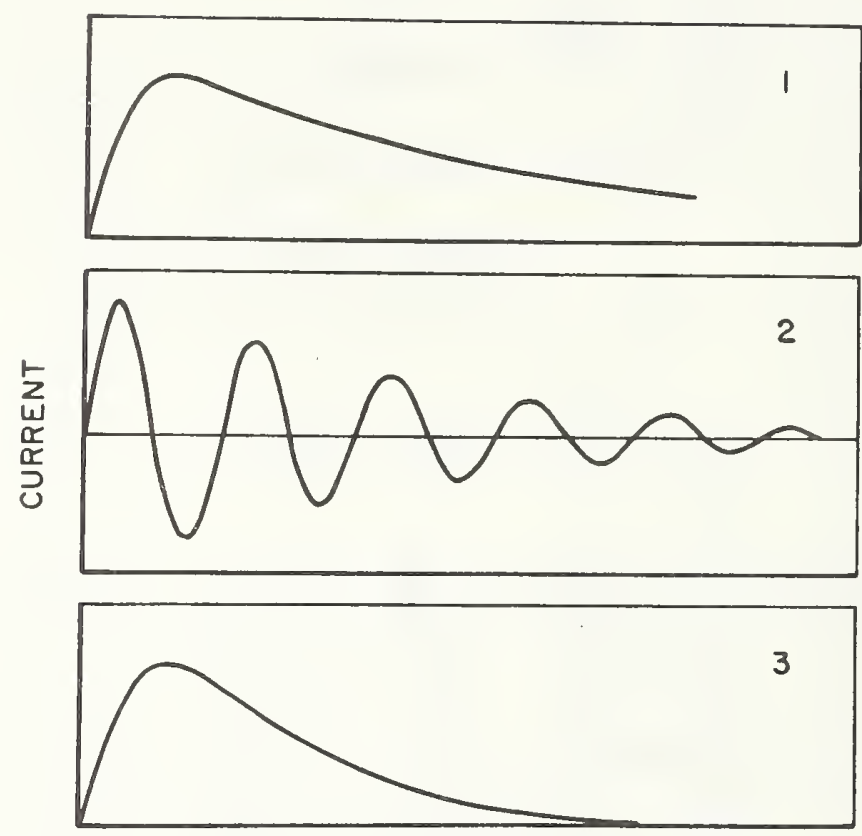

TIME

Figure 2. Typical discharge characteristics of a capacitor system.
(1) overdamped,
(2) underdamped,
(3) critically damped.

with the circuit oscillating at a natural angular frequency $\omega$ (defined in Table 1). In some experiments, the oscillating discharge through the specimen is avoided by switching a low resistance unit parallel to the specimen at the end of the first cycle or part of the first cycle. This scheme requires greater amount of stored energy since some of the energy, which normally would be imparted to the specimen, bypasses the specimen. 


\subsection{Consideration of Various Phenomena}

The successful design and operation of high-speed measurement systems and the meaningful interpretation of the data depend on detailed consideration of various thermal electrical, magnetic and other related phenomena. In the following paragraphs some of the important items are discussed.

\subsubsection{Specimen Stability-Gravitational}

A distinct advantage of the rapid pulse heating experiments is that the specimen does not need to have a container. However, one must be sure that the specimen remains in its original position and geometry during the entire experiment (which may be several hundred microseconds). Ultimately the gravitational forces will cause the specimen to collapse. A simple computation using the relation $\ell=0 t^{2} / 2$ (where $\ell$ is the distance, $t$ the time and $\alpha$ is the acceleration due to gravity) gives an estimate of the movement of the specimen due to gravity. The results indicate a movement of about $0.5 \mathrm{~mm}$ in $10 \mathrm{~ms}$ and $0.005 \mathrm{~mm}$ in $1 \mathrm{~ms}$. Since the experiment will be completed in less than $1 \mathrm{~ms}$, the gravitational force will have almost no effect on the measurements.

\subsubsection{Internal Magnetic Forces}

High currents through a conductor create magnetic forces which act toward the center of the conductor. The effect of these forces is especially critical for tubular conductors, for conductors with axially asymetric geometries, and for liquid conductors. 
Magnetic force on a cylindrical conductor is

$$
F=\frac{40 i^{2} L}{3 a}
$$

where $i$ is the current $(A)$, a is the radius $(\mathrm{cm})$ of the conductor, $L$ the length $(\mathrm{cm})$ of the conductor, and $F$ is the force $(\mu N)^{1}$. The corresponding magnetic pressure $(\mathrm{Pa})$ is given by

$$
P=\frac{0.2 i^{2}}{3 \pi a^{2}}
$$

\subsubsection{Exte mal Magnetic Forces}

The force between two current carrying conductors is expressed by

$$
F=\frac{\mu i_{1} i_{2} L}{5 r}
$$

where, $\mu$ is permeability (in vacuum, $\mu=1$ ), $i_{1}$ and $i_{2}$ are the currents (A) in the two conductors, $\mathrm{L}$ is the length $(\mathrm{cm})$ of the conductor, $\mathrm{r}$ is the separation $(\mathrm{cm})$ of the conductors and $\mathrm{F}$ is the force $(\mu \mathrm{N})$. According to the above equation, force per unit length $(\mathrm{cm})$ between two conductors carrying the same current placed in vacuum becomes:

$$
\frac{F}{L}=\frac{i^{2}}{5 r}
$$

\footnotetext{
$1_{1 \mu N}=0.1$ dyne

${ }^{2} \mathrm{~Pa}=1 \mathrm{~N} \cdot \mathrm{m}^{-2}$
} 
It may be seen. that forces resulting from currents in conductors are proportional to the square of the current. For rapid pulse experiments, where high currents (of the order of $10^{4}$ to $10^{6} \mathrm{~A}$ ) are involved, consideration of these forces become very important for the mechanical stability of the overall system as well as of the specimen.

\subsubsection{Skin Effect}

The skin effect, which is caused by changing currents in a circuit, alters the resistance of the specimen or other elements in the circuit and creates temperature nonuniformities in the specimen.

For a cylindrical conductor the skin effect is a function of the following parameter:

$$
x=2 \pi \alpha \sqrt{\left(2 \mu f / 10^{9} \rho\right)}
$$

where, $\alpha$ is the radius of the conductor $(\mathrm{cm}), f$ the frequency of current $(\mathrm{Hz}), \rho$ is the electrical resistivity of the conductor $(\Omega \cdot \mathrm{cm})$, and $\mu$ is the permeability of the conductor (for non-magnetic materials $\mu=1$ ). It may be noted that for a given geometry and frequency, the skin effect is greater for a good conductor than for a poor conductor.

In general, the contribution of the skin effect in a specimen is expressed in terms of the ratio of its electrical resistance at the frequency in question to that under d.c. conditions. Results on the resistance ratio in terms of the parameter $x$ in Equation (6) are given in the literature [8]. The contribution of the skin effect may be neglected for experimental conditions which yield a value of $\mathrm{x}$ less than 0.5 (this corresponds to a value of 0.03 percent for the resistance ratio). 


\subsubsection{Temperature Distribution in the Specimen}

Heat transfer by conduction and convection are relatively slow processes in comparison with submillisecond heating. Thus, they do not affect the temperature distribution in the specimen provided that the specimen is at a uniform temperature just before the start of pulse heating. Heat transfer by radiation may be considerable at high temperatures (above $3000 \mathrm{~K}$ ), especially for thin wires and strips. However, for a specimen having a diameter of the order of a few millimeters radiation heat loss (up to $6000 \mathrm{~K}$ ) in submillisecond regimes may not be significant (less than 1 percent). However, even though the energy loss may be insignificant, the radiation heat transfer creates a radial temperature gradient in the specimen which may create nonuniformities in the electric current density in the specimen.

The major source of temperature nonuniformities in the specimen during rapid pulse heating is likely to be the hot spots or zones that result from material inhomogeneities or geometrical nonuniformities in the specimen. Their effect becomes particularly pronounced and amplified in a specimen which has a positive coefficient for its electrical conductivity.

If the specimen geometry is not properly selected, skin effect may also be a serious source of temperature gradients. 


\subsubsection{Evaporation}

In general, the rate of evaporation from surfaces into vacuum at high temperatures may be expressed as

$$
m(T)=A e^{-E / k T}
$$

where, $\mathrm{T}$ is temperature, $\mathrm{A}$ is a constant for a given surface, $\mathrm{E}$ is the energy of evaporation, and $k$ is the Boltzmann constant. Evaporation from solids and liquids at high temperatures increases very rapidly with temperature and becomes an important phenomena to be considered in the design and operation of measurement systems. The above equation does not apply in cases where the specimen vapor pressure is high (causing multiple collisions among the molecules) or where an inert gas is used in the experiment chamber. In such cases, the evaporation mechanism is complex and it is difficult to estimate the amount of specimen evaporation.

Evaporation from the specimen affects the measurement mainly in two ways: first, by contributing to the energy loss from the specimen, and second, by disturbing the medium between the specimen and the optical instruments, such as pyrometers, cameras, etc. The effect of evaporation may be reduced by confining the specimen in a transparent solid or liquid media or in a pressurized gas. Confinements in solid or liquid media creates other problems that stem from chemical reactions and heat conduction. In most cases, the use of an inert gas under pressure may be the optimum choice. The high pressure gas 
will reduce the evaporation and will confine the vapor to a thin boundary layer. The thickness of the boundary layer is governed by the diffusion of specimen vapor in the surrounding gas and it is proportional to the square root of the product of the diffusion coeffieient and the time (experiment duration).

\subsubsection{Electrical Breakdown in Gases}

As it was discussed earlier, in order to suppress evaporation at high temperatures, the specimen may be placed in a gaseous environment.

The high voltage holding characteristics of a gas is generally expressed by the Paschen's Law, which states that the breakdown vol tage is a function of the product of the gas density $(\omega)$ and the separation between the electrodes (d), that is $E=f(\omega d)$. Thus, for a given geometrical configuration, breakdown voltage increases with increasing gas density. This implies that breakdown voltage increases with increasing gas pressure and decreases with increasing temperature. Using the perfect gas 1 aw, it may be seen that the conditions of the gas (from electrical breakdown standpoint) at $1500 \mathrm{~K}$ and at $0.5 \mathrm{MPa}$ ( 25 atm.) pressure ${ }^{1}$ is about the same as that at room temperature and $0.1 \mathrm{MPa}$ ( 1 atm.).

$\overline{1_{0.1} \mathrm{MPa}=1}$ bar $\simeq 1$ atm. 
Experimental studies, as summarized in [9], have shown that the breakdown voltage in aIr at $0.1 \mathrm{MPa}$ ( 1 atm.) over a 5 man gap is about $17 \mathrm{kV}$. When pressure $1 \mathrm{~s}$ increased to $1 \mathrm{MPa}$ ( 10 atm.), the breakdown voltage is about $150 \mathrm{kV}$. Further increase of pressure to $4 \mathrm{MPa}$ ( 40 atm.) causes the breakdown voltage to increase to about $330 \mathrm{kV}$.

The above are for conditions where the gas is electrically neutral before the breakdown. Under the conditions where there are free electrons present in the medium and the gas is partlally lonized the situation is completely different, that 1s, the breakdown voltage 1 s very low.

\subsubsection{Ionization}

The energy necessary for ionization may be given to an atom by an electron impact, the impact of a positive ion, by the absorption of radiant energy, or the gas may become so hot as to lonize the atoms thermally by the collisions of neutral atoms. These processes may occur elther singly or in combination. It is generally belleved that in gas discharges the predominant mechanism of Ionization is by electron collistons. The ionization potential of gases is an important parameter to consider in the selection of the proper environment for the specimen. For the Inert gases, the Ionization potential decreases with increasing atomic number. Helium has the highest Ionization potential (24.6 ev); the values are $21.6 \mathrm{ev}$ for neon and $15.8 \mathrm{ev}$ for argon. 


\subsubsection{Thermionic Emission}

Thermionic emission from the specimen may have several undesirable effects. It may alter the conditions of the gas, thereby causing a parallel path for the electrical current and generation of random discharges. It may alter the true voltage across the potential probes, causing measurement errors. In addition, energy loss from the specimen by thermionic emission may become appreciable at high temperatures. Thermionic emission in vacuum may be expressed as

$$
i=\bar{A} T^{2} e^{-\frac{e \phi}{k T}}
$$

where, $\mathrm{T}$ is the specimen temperature, e the electronic charge, $\mathrm{k}$ the Boltzmann constant, $\phi$ the work function, and $\bar{A}$ is a parameter different for different materials. For metallic surfaces $\bar{A}$ is approximately $60 \mathrm{~A} \cdot \mathrm{cm}^{-2} \cdot \mathrm{K}^{-2}$ and for oxides it may be in the range $1 \times 10^{-8}$ to 10 . The quantity $\phi$ may be in the range 2 to $5 \mathrm{~V}$ both for metals and oxides.

\subsubsection{Other Phenomena}

Thermal Expansion: A material expands (due to thermal effects) approximately with the speed of sound in that material. In order to avoid complications (practical and theoretical), it is advisable to limit the heating rate of the specimen to a value that allows the free expansion of the specimen at any time during the rapid heating period. The speed of sound in most solid materials is of the order of a few mllimeters per microsecond. 
Thermoelectric Effects: The Thomson effect is probably the most significant the rmoelectric effect in rapid heating experiments. Its contribution (absorption or evolution of heat) occurs only at the extreme ends of the specimen (near the clamps) where sharp temperature gradients exist. 


\section{Measurement of Experimental Quantities}

\subsection{Power Measurements}

The most frequently needed quantities in thermodynamic experiments are power absorbed by the specimen and specimen temperature. Absorbed power is determined from measurements of imparted power and power losses. In experiments in which the specimen is heated resistively, imparted power may be obtained from measurements of current through the specimen and voltage across the specimen. In capacitor discharge experiments, the only significant power loss from the specimen is that due to thermal radiation. In most cases, this loss comprises less than one percent of Imparted power. Therefore, a correction based on an estimated emittance value is generally considered to be satisfactory.

Accurate measurement of current and voltage in capacitor discharge experiments is a difficult task. This difficulty stems primarily from the high degree of interaction (due to electromagnetic fields) between the signal carrying circuits and the main discharge circuit. Since the effect of this interaction is proportional to the quantity $\mathrm{d} i / \mathrm{dt}$ (where $i$ is current and $t$ is time), increasing difficulties with increasing discharge speeds are encountered.

The state of the art of current measurement techniques is more advanced than that for voltage. The coaxial shunt, shown schematically In Figure 3, has the feature of eliminating, to a large extent, the effect of induced voltages in the signal carrying circuit. This is accomplished by having the resistance element in tubular form and by placing one of the 
vol tage probes along the axis of the tube. Because of radial symmetry, the net electromagnetic force and the net induced voltage in the probe are therefore zero. Inaccuracy in current measurements with this scheme is estimated to be approximately $1-2$ percent. Due to the skin effect in the resistance element, this inaccuracy may increase at very high oscillatory discharge frequencies (above $1 \mathrm{MHz}$ ).

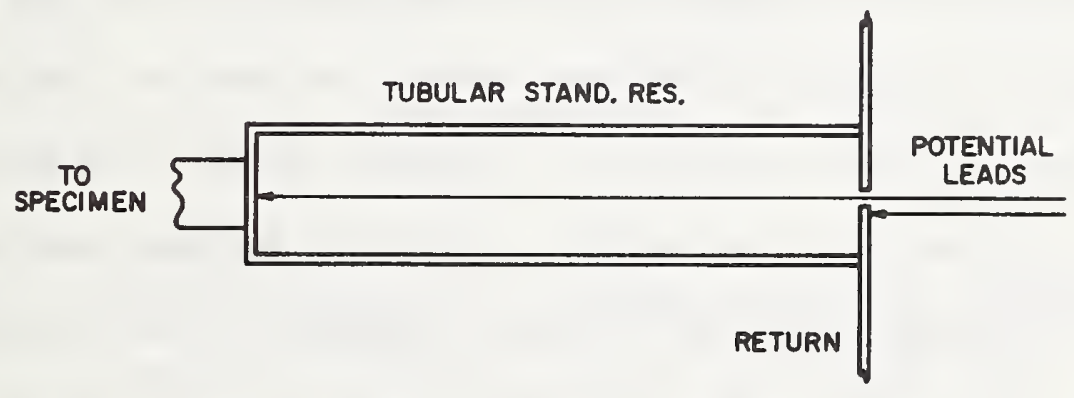

Figure 3. Schematic diagram of a tubular standard resistance used in capacitor discharge experiments.

Several methods have been used to measure voltage across the specimen during a discharge experiment. However, as of now, no practical and accurate technique has been developed. The most frequently used technique is based on measurements with a voltage divider. The divider ratio is dependent upon the voltage across the specimen and the input voltage rating of the recording instrument. Such a measurement scheme is subject to errors due to induced voltages in the measuring circuit. 
The attempts that have been made to eliminate this error or to reduce it to a negligible level may be classified in two categories: (a) by extensive shielding of all the measuring circuits, and (b) by compensating for all the induced voltages. The first method is not satisfactory because there are always portions of the circuit (those near the specimen) that cannot be properly shielded. The second method, in principle, is more promising. Several different types of compensation (mathematical and physical) may be used. Mathematical compensation is based on recording the gross voltage signal (sum of true and induced components) in addition to current through the specimen, and adjusting the voltage data (with the use of a computer) after the experiment to bring it in phase with the current. The final voltage obtained by this approach represents the true resistive component across the specimen. The same result may be accomplished by physical compensation using an adjustable coil placed in series with the voltage divider circuit. The function of the coil is to cancel erroneous voltage during the discharge by generating an induced voltage equal in magnitude but opposite In sign to that induced in the measurement circuit and the specimen. Equality between the erroneous induced voltage and the generated compensating voltage may be established by rotating the coil (by trial and error) until voltage and current traces are in phase with each other. A schematic diagram of a voltage measuring circuit based on a physical compensation technique is shown in Figure 4. An additional improvement 


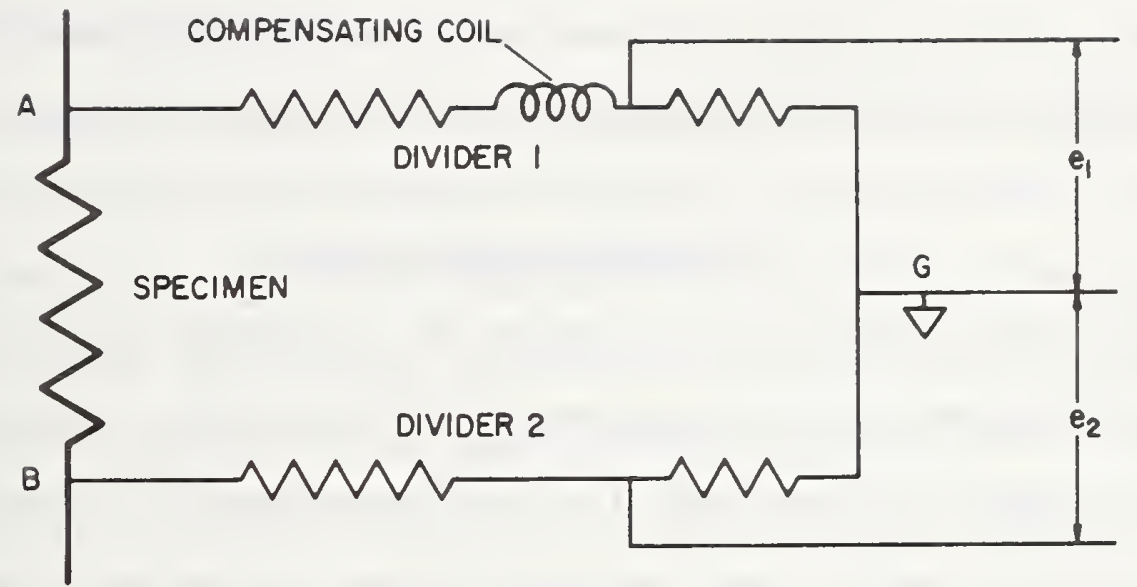

Figure 4. Electrical circuit of a voltage measuring technique utilizing two dividers and a compensating coil

incorporated in the circuit is the use of two voltage dividers. The signals corresponding to voltages $e_{1}$ and $e_{2}$ are applied differentially to an oscilloscope. The true voltage across the specimen is proportional to the quantity $\left(e_{1}-e_{2}\right)$. The advantage of the two-voltage-divider system is that it allows measurement of specimen voltage even though both terminals of the specimen may be at a high potential with respect to ground. This provides flexibility (in the grounding procedure) in the design of the main discharge circuit. Inaccuracy in voltage measurements with proper compensation techniques is estimated to be approximately three percent. 
In recent years, techniques based on electro-optical and magnetooptical effects for the measurement of pulse voltages and currents have been explored. The scheme that utilizes the optical attenuation in a Kerr cell due to high voltages across the two electrodes shows great promise for accurate measurement of pulse high voltages.

\subsection{Temperature Measurements}

There are no commercial instruments or established techniques for accurate high temperature measurements with microsecond resolution. Several attempts have been made for such measurements in connection with research on shock waves, exploding wires, and pulsed plasmas. They are generally based on photoelectric or photographic detection of the specimen radiance. Conversion of the radiance to temperature is accomplished by calibrating the detector with reference radiances before and/or after the transient experiments.

The fact that calibration and transient experiment are not made simultaneously may introduce uncertainties. For example, in the case of photomultiplier tube detectors, fatigue, instability, or the effect of high magnetic fields during a discharge may invalidate the calibration. In the case of photographic films, variations in emulsion and development may introduce similar uncertainties. To avoid these difficulties it is necessary to develop systems in which the detector is calibrated during the transient experiment. In photoelectric systems this may be accomplished using a high-speed chopper which allows the alternate exposure of the detector to radiation from the unknown 
and from a reference. In photographic systems, no chopping is necessary and it suffices to photograph simultaneously one or more reference radiances in addition to the unknown on the same film during the transient experiment.

Another alternative that avoids the use of chopping schemes, is the development of photoelectric or similar detectors which are very stable and whose behavior is invariant under transient electromagnetic fields. Recent developments in the area of solid-state detectors show considerable promise. Their submicrosecond response characteristics are attractive and their stability and reproducibility under relatively slow experiments (millisecond resolution) have been satisfactory. However, no careful study has yet been made regarding their stability and behavior under intense short radiation pulses and strong electromagnetic fields. Most solid-state detectors developed so far have a maximum response in the near infrared and infrared regions. In some cases, the response curve does not show a sharp decrease toward the visible region and extends to about $400 \mathrm{~nm}$. These characteristics are sufficient to satisfy some of the requirements of a microsecond resolution temperature measurement system. However, as it was mentioned above, their behavior under intense short radiation pulses and under high electromagnetic fields needs to be studied.

Consideration of the photon energy requirements for the satisfactory operation of a detector suggests that solid-state detectors can be used for temperature measurements (with microsecond resolution) above 
about $2500 \mathrm{~K}$. By optimizing the effective wavelength, the exposure times can be further reduced. Also, selection of the bandwidth of radiation impinging on the detector contributes to the satisfactory response of the detector. The bandwidth of the filters used for this purpose may be in the range 10 to $50 \mathrm{~nm}$.

Temperature measurements based on radiance measurements at one wavelength are possible for cases where the specimen approximates blackbody conditions or the normal spectral emittance of the specimen at the effective wavelength of the instrument is known. If these conditions are not met, the alternative may be to perform radiance measurements simultaneously at two wavelengths. This provides one to obtain the specimen's true temperature under the assumption that the ratio of specimen emittance at the selected wavelengths is independent of temperature. This approach seems to have promise in measuring the temperature of rapidly heating solids and especially liquids at temperatures above $2500 \mathrm{~K}$ in microsecond-resolution experiments. However, it has the disadvantage of being insensttive compared to a monochromatic pyrometer, and it is subject to a systematic error if the ratio of emittances at the selected wavelengths varies with temperat ure. 


\subsection{Other Measurements and Diagnostic Techniques}

In addition to the measurement of imparted power to the specimen and specimen temperature, time-resolved measurements of other quantities may be required during a pulse heating experiment. For example, the pressure, shape, expansion, and density of the specimen may be needed.

Transient pressure measurement techniques have been developed primarily in connection with shock tube research. In general, they utilize transducers whose operation is based on piezoelectric or piezoresistive effects.

For the measurement of quantities related to specimen geometry, high-speed photography and flash $\mathrm{x}$-ray techniques may be employed. However, in some cases considerable discrepancies have been observed between the results obtained simultaneously by the two methods. In general, results from $x$-ray data are considered to be more reliable in measurements related to the diameter of an expanding specimen.

It is important to have diagnostic techniques to ascertain the specimen behavior in submillisecond-duration experiments as well as the electrical and optical conditions in the experiment chamber. High-speed photography is used for both diagnostic and measurement purposes. Improvements in films and optical comonents, including utilization of electro-optical devices and pulsed lasers, have enhanced the contribution of high-speed photography to capacitor discharge experiments. 
Techniques for modulating optical radiation play an important role in high-speed experiments not only in connection with temperature measurements but also in other optical studies as in high-speed photography, spectroscopy, and interferometry. They may be divided into two general classes: (a) mechanical modulation, and (b) electrooptical or magneto-optical modulation. Most commonly used mechanical modulators are disc-shaped, choppers, and turbine mounted mirrors; they may be used for modulating 1 ight to $10^{4}$ and $10^{7} \mathrm{~Hz}$, respectively. For higher frequencies (up to $10^{9} \mathrm{~Hz}$ ) electro-optical modulators (Kerr cel1, etc.) may be used. However, as of the present time, very 11ttle accurate work has been done to assess the operational characteristics of electro-optical modulators, such as transmission stability, and behavior under varying intemal and external parameters.

Generation of short-duration light pulses, which may be needed in certain high-speed experiments, may be accomplished either by modulating a steady Iight source or by having a pulsed light technique. The latter has the advantage of delivering higher intensities. Flash lamps were used in the past for this purpose. However, advances in pulsed lasers have overshadowed all other pulsed 1ight sources. They are capable of providing intense monochromatic light, and their pulse duration may be made as short as a few picoseconds. 


\subsection{Recording of Experimental Quantities}

The ultimate utility of a measurement system lies in the proper recording of the experimental quantities. One of the major reasons for the delay in the development of high-speed thermodynamic and related measurement techniques has been the difficulties encountered in accurate recording of the signals.

The first truly high-speed recording of quantities was realized with the development of modern oscilloscopic techniques. Because of the very fast response characteristics (as low as nanoseconds), oscilloscopes were used for a wide range of experimental conditions, requiring from millisecond to nanosecond resolution. However, they have limitations which stem primarily from their relatively poor recording accuracy. In general, under optimum conditions oscilloscopic recording inaccuracy can hardly be less than one to two percent of full scale. In special cases where pulses to be recorded are of rectangular or trapezoldal shape, accuracy may be improved by suppressing the major portion of the pulse and recording only the remainder of the signal. Since oscilloscopic recording (on film) is in analog form, additional effort is required to reduce the information to numeric (digital) form using micrometer microscopes or similar reading instruments.

Advances in the electronics and computer flelds during the last decade have made the development of accurate high-speed digital recording systems a possibility. Such systems generally consist of a multiplexer, an analog-to-digital converter, a memory, pertinent 
interfacing equipment, and an output device (such as a magnetic tape deck, a teletypewriter, or a computer). For systems where recording resolution must be less than a few microseconds, the multiplexer is omitted and separate analog-to-digital converters in conjunction with sample-and-hold amplifiers are used for each channel (experimental quantity).

With the presently available electronic components and technology it is possible to build a digital data acquisition system capable of digitizing and recording simultaneously a set of several experimental quantities about every $0.5 \mu$ with a full-scale signal resolution of about 0.1 percent under optimum conditions. 


\section{Special Considerations in Relation to $\mathrm{UO}_{2}$}

The presentation and discussion of various phenomena and experimental conditions in the previous sections were based on the fact that the specimen was a good electrical conductor, meaning that it could have been resistively heated from room temperature up to the desired high temperature by discharging a capacitor bank through it.

Almost all oxides are either nonconductors or poor conductors at room temperature. However in a number of cases, their electrical conduction properties increase rapidly with increasing temperature. Uranium dioxide exhibits such a behavior. In this section, techniques for performing transient measurements on $\mathrm{UO}_{2}$ and the problems associated with such methods are discussed.

\subsection{Heating of $\mathrm{UO}_{2}$}

Electrical conductivity is the most important property in considering the resistive self-heating of the specimen. The electrical conductivity of uranium dioxide depends strongly on its composition, namely on the oxygen to uranium ratlo. The avallable literature on electrical conductivity of uranium dioxide is not conclusive, but it is sufficient to give a general idea as to the magnitude and the range of values. For the stoichiometric composition, the electrical conductivity at room temperature is of the order of $10^{-7} \Omega^{-1} \cdot \mathrm{cm}^{-1}$, and increases to about $10^{-3} \Omega^{-1} \cdot \mathrm{cm}^{-1}$ for oxygen to uranium ratio of 2.001 [10]. The data reported in the literature for the oxygen deficient cases are very few. The avallable results indicate that at 
room temperature electrical conductivity is of the order of $10^{-4}$ $\Omega^{-1} \cdot \mathrm{cm}^{-1}$ for the case where the oxygen to uranium ratio is 1.990 [11]. It seems that as the temperature is increased the differences in electrical conductivity due to differences in composition are reduced. For discussion and general estimative purposes, one may consider the values $1 \Omega^{-1} \cdot \mathrm{cm}^{-1}$ at $1500 \mathrm{~K}$ and $50 \Omega^{-1} \cdot \mathrm{cm}^{-1}$ at $3000 \mathrm{~K}$ [12].

From the above figures, it may be seen that $\mathrm{UO}_{2}$ becomes a fairly good conductor at temperatures above $1500 \mathrm{~K}$. Therefore, one may consider a two-stage heating procedure, in which the specimen is heated slowly up to about $1500 \mathrm{~K}$ and above that temperature it is heated rapidly.

The first-stage slow heating may be accomplished either by resistive self-heating or by external heating using a laser or a conventional furnace arrangement. The slow resistive self-heating method may not be desirable because it causes the establishment of axial and radial temperature gradients in the specimen. For similar reasons, laser heating is not suitable. Problems with electrical interference may preclude the use of direct or indirect $\mathrm{RF}$ heating. It seems that a conventional tubular furnace arrangement may be the best solution to heat the specimen to about $1500 \mathrm{~K}$.

Once the specimen is at a constant temperature at about $1500 \mathrm{~K}$, the second-stage rapid heating up to about $6000 \mathrm{~K}$ may be accomplished by discharging a capacitor bank through the specimen, as was described in the earlier sections of this report. The melting point of $\mathrm{UO}_{2}$ is 
about $3130 \mathrm{~K}$, thus the rapid heating will cover part of the solid phase as well as the 11quid phase.

Uniformity in specimen temperature distribution at the end of the first-stage heating is a very important consideration. If temperature gradients or other nonuniformities exist in the specimen before the second-stage heating starts, these nonuniformities are $11 \mathrm{kely}$ to be amplifled and cause specimen fallure during the rapld heating period.

$\mathrm{A} \mathrm{UO}_{2}$ specimen, $5 \mathrm{~mm}$ in diameter and $15 \mathrm{~mm}$ in length, has an electrical resistance of about $7.6 \Omega$ at $1500 \mathrm{~K}$ (based on a conductivity value of $1 \Omega^{-1} \cdot \mathrm{cm}^{-1}$ ). Considering the values of $1 \mu H$ for the inductance and $100 \mu \mathrm{F}$ for the capacitance of a typical discharge circuit, it may be seen from the relation in Table 1 that the discharge w111 correspond to the "overdamped" case, and the current waveform will resemble the one shown In Insert 1 of F1gure 2. The waveform of current and voltage may change as the resistance of the sample decreases from $7.6 \Omega$ at $1500 \mathrm{~K}$ to about $0.15 \Omega$ at $3000 \mathrm{~K}$.

Energy required to heat the $\mathrm{UO}_{2}$ specimen from 1500 to $6000 \mathrm{~K}$ is estimated based on the enthalpy data given in the 11terature [13] for temperatures up to the melting point, the heat of fusion also given in the literature [14], and assumed constant heat capacity of 11 quid $\mathrm{UO}_{2}$ equal in value to that of solid $\mathrm{UO}_{2}$ near the melting point. The results yield a value of about $800 \mathrm{~kJ} \cdot \mathrm{mol}^{-1} \cdot \mathrm{A} \mathrm{UO}_{2}$ specimen $5 \mathrm{~mm}$ in diameter and $15 \mathrm{~mm}$ in length weighs about $3.12 \mathrm{~g}(0.0115 \mathrm{~mol})$. Thus, energy required to heat the $\mathrm{UO}_{2}$ specimen from 1500 to $6000 \mathrm{~K}$ is about $9 \mathrm{~kJ}$. 
A capacitor bank (eight capacitors, $20 \mathrm{kV}, 15 \mu \mathrm{F}$ each) capable of storing $24 \mathrm{~kJ}$ should be sufficient to provide the required energy. This allows for a 60 percent energy loss in the switch and other parts of the circuit during the discharge.

The dimensions of the specimen given above are an upper limit. A shorter specimen may be needed in order to increase the electric field and the heating rate. In other cases, a smaller diameter may be required in order to reduce the energy requirements because part of the energy may be dissipated either in a series resistance or in a current shunt (crowbar switch).

\subsection{Various Phenomena Related to UO 2}

In Section 3.2 of this report, various phenomena that had to be considered in the design and operation of high-speed measurement systems were discussed on a general basis. In the present section, the contribution of these phenomena in the specific case of $\mathrm{UO}_{2}$ as the specimen are summarized. For the descriptions of the phenomena, for the pertinent equations, and for relevant discussions one may refer to Section 3.2. In the rest of this section, duration of the experiments is considered to be in the range 10 to $1000 \mu \mathrm{s}$. The various phenomena are evaluated to correspond to the most unfavorable case.

Specimen Stability-Gravitational: Movement of the specimen due to gravitational forces is estimated to be less than 0.01 mm in $1000 \mu s$. 
Internal Magnetic Forces: Internal magnetic forces create pressures that act toward the axis of the specimen. This discourages the use of tubular specimens; however, specimens in the form of rods should not be significantly affected by these pressures.

External Magnetic Forces: The mechanical stability of the specimen will depend to a very large extent on the balance of external magnetic forces. To achieve this, a high degree of axial symetry is required between the specimen and the external current paths. An accurate coaxial configuration between the specimen and the current return path will minimize the effect. An eccentricity of $1 \mathrm{~mm}$ between the specimen axis and that of the current return cylinder is likely to produce magnetic forces sufficient to move the specimen several millimeters In 1000 us. Since, the alignment accuracy can be better than $1 \mathrm{~mm}$, the contribution of this effect can be made small. However, experimental studies of pulse discharges in metals have shown that the specimen becomes unstable in times much less than 1000 us. From the results of such studies, the time available for the measurements in the liquid phase is likely to be in the range 10 to $20 \mu \mathrm{s}$. This corresponds to an average heating rate in the range 150 to $300 \mathrm{~K}^{\prime} \mathrm{us}^{-1}$ for heating the specimen from 3000 to $6000 \mathrm{~K}$. An electrical field in the range 5 to $10 \mathrm{kV} \cdot \mathrm{cm}^{-1}$ will be required for these high heating rates. 
Skin Effect: Considering that the specimen diameter is $5 \mathrm{~mm}$ and that the experiment duration is $10 \mathrm{\mu s}$, the skin effect is estimated to be less than 0.03 percent at $3000 \mathrm{~K}$; this value indicates the ratio of the electrical resistance at the frequency in question to that under d.c. conditions. At lower temperatures, the effect is even less, since the electrical conductivity is less. No data on electrical conductivity of liquid $\mathrm{UO}_{2}$ were found in the literature, thus it was not possible to estimate the skin effect at temperatures above the melting point. However, it may be said that, in order for the skin effect to be 1 percent at $6000 \mathrm{~K}$ it is necessary for the electrical conductivity at $6000 \mathrm{~K}$ to be 5000 times that of at $3000 \mathrm{~K}$; which is a very large factor for changes in electrical conductivity in liquids. Temperature Distribution in the Specimen: In submillisecond duration experiments heat transfer by conduction is very small, thus they do not affect the temperature distribution in the specimen. Radiation heat transfer may become appreciable at high temeratures. Considering a $\mathrm{UO}_{2}$ specimen $5 \mathrm{~mm}$ in diameter which is heated from room temperature to $6000 \mathrm{~K}$ in $1000 \mu \mathrm{s}$, heat loss by thermal radiation is less than 0.5 percent of the total power input to the specimen. The above computation is based on a hemispherical total emittance of unity, which means that in the real case heat loss is even less. By introducing a correction term based on an assumed emittance, it is possible to reduce the uncertainty in the measured property (heat capacity) due to the heat loss correction to about 0.1 percent or less. As it was mentioned 
earlier, the major source of temperature nonuniformities in the specimen is likely to be the hot channels or zones that result from material inhomogenelties or geometrical nonuniformities. These are likely to be pronounced in the case of $\mathrm{UO}_{2}$ specimens in contrast to metallic specimens. A major contributing factor to this is the positive coefficlent of the electrical conductivity of $\mathrm{UO}_{2}$. This means that as a hot zone is developed in the specimen the likely trend will be the amplification of this zone in terms of temperature with increasing time (as long as electrical current flows through the specimen) causing nonuniform heating similar to that causes breakdown of dielectric materials in high electrical fields. Thus, it is of utmost importance to have specimens with compositional homogeneity and geometrical uniformity.

Evaporation: The vapor pressure of $\mathrm{UO}_{2}$ is about $5 \mathrm{kPa}(20.05 \mathrm{~atm}$ ) at $3000 \mathrm{~K}$, and based on an extrapolation of the low temperature data it is estimated to be of the order of $10 \mathrm{MPa}(\sim 100 \mathrm{~atm} \cdot)$ at $6000 \mathrm{~K}$ [13]. Because of the high vapor pressure, it will be necessary to perform the experiments with the specimen in a pressurized environment. An inert gas, such as argon or helium, may be used. Based on the assumption that the ambient pressure must be at least about 15 times the vapor pressure of the specimen at any given temperature, it may be possible to conduct experiments at about $0.1 \mathrm{MPa}$ ( 1 atm.) pressure near the melting point of $\mathrm{UO}_{2}{ }^{\circ}$ However, the pressure may have to be increased with increasing temperature: $5 \mathrm{MPa}(\sim 50 \mathrm{~atm}$ ) at $4000 \mathrm{~K}, 50 \mathrm{MPa}$ 
( $500 \mathrm{~atm}$ ) at $5000 \mathrm{~K}$ and $150 \mathrm{MPa}(\sim 1500 \mathrm{~atm}$ ) at $6000 \mathrm{~K}$. External pressure will reduce the evaporation and thus will confine the vapor to a thin boundary layer. Under these conditions, it is likely that the boundary layer will be transparent enough not to interfere with the optical temperature measurements. Under the above conditions, heat loss due to evaporation will be negligibly small, less than 1 $\mathrm{J} \cdot \mathrm{cm}^{-2}$. The evaporative cooling effect on the specimen surface may be of the order of a few degrees.

Ionization, The rmionic Emission and Electrical Breakdown: Since the effects of these are interrelated they are discussed together. Electrical breakdown in gases may be of the order of 20 to $30 \mathrm{kV} \cdot \mathrm{cm}^{-1}$ at normal temperature and pressure. Since breakdown voltage increases with gas pressure, voltage breakdown may not be a serfous consideration by itself provided that there is no gas ionization or thermionic emission. If the $\mathrm{UO}_{2}$ specimen has to be preheated to about $1500 \mathrm{~K}$ before pulsing it to higher temperatures, the preheating may present a serious problem if gas ionization or thermionic emission gives an electrical conductivity in the gas sufficient to cause shunting of an appreciable current around the specimen. Experimental work in connection with exploding conductor research and thermal measurements on metallic specimens has not indicated any electrical breakdown in gases surrounding the specimen up to about $8000 \mathrm{~K}$ when the gas pressure is high. However, because of lack of data on $\mathrm{UO}_{2}$ at temperatures above its melting point, it is not possible to 
predict the effect of thermionic emission and other related effects on voltage breakdown in the gas surrounding the specimen. Pulse heating of $\mathrm{UO}_{2}$ will require much higher electric fields than that required for metals because of the high electrical resistivity of $\mathrm{UO}_{2}$ relative to that of metals.

\subsection{Measurements on $\mathrm{UO}_{2}$}

Measurements of the electrical quantities (current through the specimen and potential across the specimen) are, to a large extent, independent of the specimen material. The measurement techniques were summarized in Section 4.1. From the accuracy point of view, it is desirable to have the separation between the potential probes as large as possible, however, considerations due to resistance, energy and pressure are likely to restrict the length of the $\mathrm{UO}_{2}$ specimens to 1 to $2 \mathrm{~cm}$. It is estimated that current can be measured with an inaccuracy of about 2 percent and potential across the specimen with an inaccuracy of about 3 percent. This means that the inaccuracy of power measurements is likely to be within 5 percent if gas conduction is small.

Measurements of the specimen temperature depend extensively on the specimen and its reaction with its environment. Some of the problems that arise from this were discussed in Section 5.2 for $\mathrm{UO}_{2}$.

From instrumentation point of view, studies have demonstrated the feasibility of constructing a pyrometer capable of performing temperature 
measurements in the range 2500 to $6000 \mathrm{~K}$ once or twice every microsecond. The pyrometer will have two independent optical channels in order to perform radiance measurements at two wavelengths. This scheme will yield the specimen temperature from the measurements of two radiance temperatures at the two wavelengths. Based on the detector (solidstate) characteristics, it seems that it is advantageous to select the wavelengths from the range 500 . to $1000 \mathrm{~nm}$. The effective bandwidth of the interference filters may be in the range 10 to $40 \mathrm{~nm}$.

The inaccuracy in temperature measurements contributed by the pyrometer operation alone is estimated to be about 1 percent. About the same level of uncertainty is likely to be introduced in the interpretation of measured temperatures up to about $4000 \mathrm{~K}$ increasing to twice that at $6000 \mathrm{~K}$. Thus, it may be possible to measure specimen temperature with an inaccuracy of about 2 percent in the range 2500 to $4000 \mathrm{~K}$ increasing to about 3 percent at $6000 \mathrm{~K}$.

Techniques for recording experimental quantities were discussed in Section 4.4. It may be summarized by saying that it is within the state-of-the-art to construct a high-speed digital data acquisition system capable of digitally recording a set of several quantities simultaneously every 0.5 us with a full-scale signal resolution of about 0.1 percent under optimum conditions, but may be considerably worse when large nonlinear variations in the signals occur. 
It is of importance to consider protecting the measuring and recording instruments from high electro-magnetic fields resulting from the capacitor discharges. Proper shielding will reduce the interference of the fields on the measuring instruments and the signals.

From the measurements of current, voltage and temperature as a function of time during the rapld heating period it is possible to determine heat capacity as a function of temperature using the equation:

$$
c_{p}=\frac{e i-q}{n \frac{d T}{d t}}
$$

where, $e$ and $i$ are voltage and current respectively, $n$ is the amount of specimen, $d T / d t$ is the heating rate, and $q$ is the rate of total energy loss from the specimen. The quantity $q$ is usually small (not more than a few percent of imparted power) in submillisecond experiments and depending on 1 ts magnitude, efther 1 t may be neglected or accounted for by estimating the pertinent parameters. As a by-product of these experiments it is possible to obtain the electrical resistivity of the specimen.

It may be much more difficult to determine experimentally the vapor pressure of $\mathrm{UO}_{2}$ up to $6000 \mathrm{~K}$. Because of the high vapor pressure at temperatures above $3000 \mathrm{~K}$, the Langmulr method and the atomic (molecular) absorption technique camot be used. One possible approach may be based on conducting a serles of experiments with the specimen in an inert gas environment at different static pressures. 
The observation (by optical and/or electrical means) of the start of boiling and its correlation with temperature may yield vapor pressure. In the case of $\mathrm{UO}_{2}$ this corresponds to static pressures in the approximate range 0.01 to $10 \mathrm{MPa}(20.1$ to $100 \mathrm{~atm}$ ) to cover the range from the melting point to $6000 \mathrm{~K}$. Superheating of the liquid may occur before the boiling becomes detectable. Optical absorption by the vapor also may prevent measurement of radiation from the liquid surface and therefore may prevent the measurement of specimen temperature. The performance of preliminary experiments is essential in order to assess the applicability of this technique to vapor pressure measurements of $\mathrm{UO}_{2}$. 


\section{Summary and Conclusions}

The design of a system for thermal measurements in submillisecond duration experiments at high temperatures (2000 to $6000 \mathrm{~K}$ ) on semiconducting materials is subject to considerations of various phenomena characteristic of high voltage-high current discharges, high temperatures, and short experimental and measurement times. Item-by-item consideration of the various phenomena does indicate the presence of some surmountable problems. However, because of conflicting requirements, their combination may present some serious difficulties.

For the convenience of discussions, the measurement system may be divided into three parts: (a) the discharge and associated control equipment, (b) the specimen and the experiment chamber, and (c) the measuring and recording equipment.

The electrical circuitry for the high-voltage capacitor discharge system does not present any serious problems since it is likely that a $20 \mathrm{kV}$ capacitor bank will be sufficient for this purpose and the techniques for constructing and operating such a circuit are available.

The experiment chamber including the specimen does present some serious problems that stem from the combination of high voltages, high pressures, and high temperatures. The high vapor pressure of $\mathrm{UO}_{2}$ at high temperatures necessitates the pressurization of the chamber to reduce evaporation. Such high pressures restrict the size of the working volume which, in turn, may create problems in relation to preheating the specimen. The high voltages required for pulse heating 
the specimen may cause additional complications from electrical insulation point of view. However, systems have been constructed and operated under high pressure-high voltage conditions for rapid pulse heating (microsecond resolution) of good conductors (at room temperature) to temperatures over $6000 \mathrm{~K}$. Preheating of $\mathrm{UO}_{2}$ specimens to about $1500 \mathrm{~K}$ has been performed in experiments in which slow pulse heating (millisecond resolution) was used to heat the specimens up to about $3000 \mathrm{~K}$. Faster heating requires higher vol tages. Nevertheless, in spite of all the difficulties, it may be possible to construct an experiment chamber for performing rapid pulse heating experiments at high temperatures. Because of the high positive coefficient of the electrical conductivity of $\mathrm{UO}_{2}$, compositional inhomogeneties of geometrical nonuniformities are likely to create large temperature gradients and thermal breakdown in the specimen. However, by careful preparation of the specimens their effect may be minimized.

The measuring equipment and the techniques which include current, vol tage, pressure, temperature, and other optical measurements with microsecond resolution are only partially developed. Temperature measurement is the most difficult one. Based on the avallable information, it seems likely that a pyrometer with microsecond resolution can be constructed for radiance temperature measurements at two wavelengths. Recording of experimental quantities can be made using oscilloscope-camera combinations, or for somewhat better accuracy and 
convenience, digital recording techniques can be utilized. It is within the state-of-the-art to construct a digital data acquisition system for recording several quantities every 0.5 us with a fullscale signal resolution in the range 0.1 to 0.5 percent. It is estimated that electrical power measurements can be made with an inaccuracy of about 5 percent and temperature measurements with an inaccuracy of 2 to 3 percent. The resultant measurements are 11 kely to yield heat capacity with an inaccuracy of about 7 percent at $3000 \mathrm{~K}$ increasing to about 10 percent at $6000 \mathrm{~K}$. The inaccuracy of vapor pressure measurements is likely to be considerably higher, however, no specific estimation is possible at this time.

Based on the discussions in this report, it may be concluded that the capacitor discharge circuitry and the measurement of the experimental quantities, although difficult, are not likely to present major problems. The major experimental problems will primarily stem from the experiment chamber and the specimen. The combined contributions of some of the problems are difficult to estimate and therefore, some preliminary experiments need to be performed to assess the applicability of the submillisecond-duration heating techniques to measurements on $\mathrm{UO}_{2}$. The preliminary experiments should yield information on:

(a) the mechanical stability of the specimen (in the solid and especially in the liquid phase) under high electromagnetic fields;

(b) specimen integrity under rapid heating conditions which includes the effects of thermal breakdown, thermal shock, disintegration that may result from rapid discharges through the specimen; 
(c) level of evaporation and its effect on optical and electrical measurements;

(d) the effect of specimen preheating furnace on gas ionization and high vol tage breakdown.

If a method can be found to heat the specimen to and through the melting phase change at moderate pressures, then it may be possible to increase the pressure sufficiently to achieve satisfactory results in the liquid range up to $6000 \mathrm{~K}$.

Positive results from the above preliminary experiments in conjunction with the development of electrical pulse power and temperature measuring equipment will suggest the feasibility of conducting thermodynamic measurements on $\mathrm{UO}_{2}$ up to $6000 \mathrm{~K}$ utilizing submillisecondduration resistive self-heating techniques. 


\section{References}

[1] Chase, W. G. and Moore, H. K., eds., "Exploding Wires", Plenum, New York, Vol. I (1959), Vol. II (1962), Vol. III (1964), Vo1. IV (1968).

[2] Lebedev, S. V., "The possibllity of using the electrical explosion of wires for investigation of metals at high temperatures", High Temperature, $\underline{6}, 150$ (1958).

[3] Dikhter, I. Ya. and Lebedev, S. V., "Measurement of the specific heat and the heat of fusion of tungsten at high temperatures by the wire explosion technique", High Temp.-High Pres., $\underline{2}$, 55 (1970)。

[4] Henry, K. W., "A technique for measuring equilibrium thermodynamic states of liquid metals at high temperatures and pressures", Rev. Sc1. Instr., 43, 1777 (1972).

[5] Martynyuk, M. M. and Karimkhodzhaev, I., "Estimation of critical temperatures of metals by the exploding wire method", Russ. J. Phys. Chem., 48, 722 (1974).

[6] Gathers, G. R., Shaner, J. W., and Young, D. A., "Experimental very high temperature liquid uranium equation of state", Lawrence Livermore Laboratory Preprint 75668 (1974).

[7] Martynyuk, M. M., Karimkhodzhaev, I., and Tsapkov, V. I., "Resistance and enthalpy of refractory exploding wires", Sov. Phys. Tech. Phys., 19, 1458 (1975). 
[8] Grover, F. W., "Inductance Calculations", Van Nostrand, New York, 1946 , p. 266 .

[9] Cobine, J. D., "Gaseous Conductors", McGraw-Hill, New York, 1941.

[10] Thermodynamic and Transport Properties of Uranium Dioxide and Related Phases, Int. Atomic Energy Agency, Vienna, 1965.

[11] Iida, S., "Electrical Properties of Non-Stoichiometric Uranium Dioxide", Jap. J. Appl. Phys., 4, 833 (1965).

[12] Bates, J. L., Hinman, C. A., and Kawada, T., "Electrical Conductivity of Uranium Dioxide", J. Am. Cer. Soc., 50, 652 (1967).

[13] Chasanov, M. G., Leibowitz, L., and Gabelnick, S. D., "High Temperature Properties of Fast Reactor Materials", J. Nuc. Mat., 49, 129 (1973-1974).

[14] Hein, R. A., Flagella, P. N., and Conway, J. B., "High-Temperature Enthalpy and Heat of Fusion of $\mathrm{UO}_{2}$ ", J. Am. Ceram. Soc., $\underline{51}$, 291 (1968). 


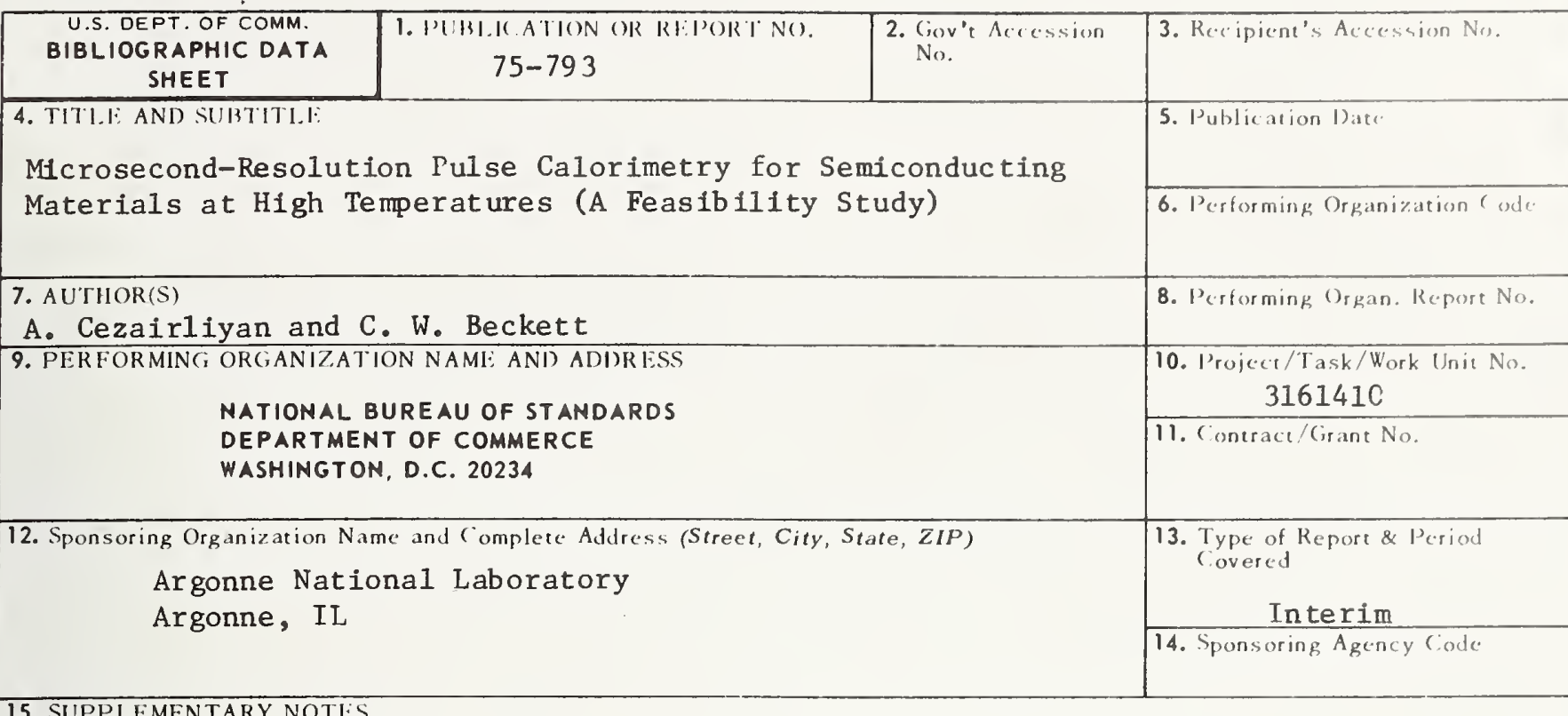

15. SUPPIEMENTARY NOTLS

16. ABSTR AC:T (A 200-word or less factual summary of most sipnificant information. If document includes a significant bibliography or literature survey, mention it here.)

A feasibility study is conducted for the applicability of microsecond-resolution pulse heating techniques to the measurement of selected thermodynamic properties of semiconducting substances at high temperatures. The method of pulse heating of the specimen using capacitor discharges is described and general design considerations, including electrical circuitry and various physical, electrical and chemical phenomena, are presented. Measurements of experimental quantities, such as current, voltage, temperature and pressure are described. The special problems in relation to measurements of heat capacity and vapor pressure of uranium dioxide in the temperature range 2000 to $6000 \mathrm{~K}$ are discussed. It is concluded that the capacitor discharge circuitry and the measurement of the experimental quantities, al though difficult, are not likely to present major problems. However, problems are likely to stem from the experiment chamber and the specimen. The nature of these problems are discussed, and the performance of some preliminary experiments are suggested.

17. KEY WORDS (six to twelve entries; alphabetical order; capitalize only the first letter of the first key word unless a proper name; separated by semicolons) Capacitor discharges; heat capacity; high-speed measurements; high temperature; thermodynamics; uranium dioxide.

18. AVAILABILITY

For Official Distribution. Do Not Release to NTIS

[] Order From Sup. of Doc., U.S. Ciovernment Printing Office Washington, D.C. 20402, SD Cat. No. C.13

E. Order From National Technical Information Service (NTIS) Springfield, Virginia 22151

\begin{tabular}{|l|c|}
\hline $\begin{array}{l}\text { 19. SECURITY CL.ASS } \\
\text { (THIS REPURT) } \\
\text { UNCL ASSIFIED }\end{array}$ & 21. NO. OF PAGES \\
\hline $\begin{array}{l}\text { 20. SF(URITY CLAASS } \\
\text { (THIS PAGE) }\end{array}$ & 22. Price \\
UNCLASSIFIED) & $\$ .75$ \\
\hline
\end{tabular}





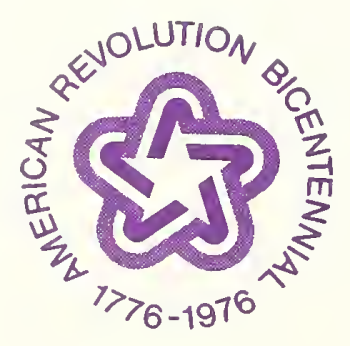

